\title{
Modelo didáctico para el perfeccionamiento de la enseñanza-aprendizaje de la geometría en la carrera de Ingeniería
}

\author{
Teaching model for the improvement of the teaching and learning \\ of geometry in engineering
}

\author{
Carlos Wilson Lizarazo Gómez \\ Máster en Educación Matemática. Profesor de Matemáticas y director del grupo investigación Génesis de la Facultad de \\ Ciencias Básicas-Universidad del Atlántico, Barranquilla, Colombia. Av. vía Puerto Colombia \\ E-mail:carlos_wilsonl@hotmail.comylizarazo@uniatlánticoedu.cu
}

Para citar este artículo: Lizarazo, C. (2016). Modelo didáctico para el perfeccionamiento de la enseñanza-aprendizaje de la geometría en la carrera de Ingeniería. Escenarios, 14 (2), p,p 31-44

DOI: http: / / dx.doi.org/10.15665/esc.v14i2.930

Recibido: julio 5 de 2016

Aprobado: 4 de 2016

\section{RESUMEN}

Este artículo presenta el uso de herramientas de informática educativa y otros recursos tecnológicos en la escuela, los cuales se han convertido en un gran aliado para el desarrollo de habilidades y destrezas en los alumnos, transformándose las mismas en recursos de apoyo para la enseñanza aprendizaje de la matemática. Lamentablemente, no se logra un acceso pleno del docente a este cambio, ya que aún se mantiene el uso de la tiza y el pizarrón. Por esta razón, en este trabajo de investigación se propone, una descripción coherente del diseño de un modelo didáctico y un procedimiento didáctico mediante el uso de software dinámico para el perfeccionamiento de la enseñanza-aprendizaje de la geometría en la carrera de Ingeniería. La investigación se fundamenta en la dialéctica materialista y toma elementos de los paradigmas cuantitativos y cualitativos, y se triangulan los resultados, concepciones y fuentes

Palabras Clave: Modelo didáctico, software dinámico, enseñanza-aprendizaje de la geometría

\begin{abstract}
This article presents the use of tools of computer education and other technological resources at the school, which have become a great ally for the development of abilities and skills in students, transforming them into supportive resources for teaching mathematics learning. Unfortunately, cannot access full teaching to this change, because that is still the use of chalk and the Blackboard. By this reason, in this work of research is proposes, a description coherent of the design of a model teaching and a procedure teaching through the use of software dynamic for the improvement of the teaching-learning of the geometry in the career of engineering. The research is based on the materialist dialectic and takes up elements of the qualitative and quantitative paradigms, and results, concepts and sources was triangulated.
\end{abstract}

Keys words: Model training, software dynamic, learning of the geometry 


\section{INTRODUCCIÓN}

Para lograr el perfeccionamiento de la enseñanza aprendizaje de la geometría en Ingenierías mecánica Guin y Trouche (1999) mencionan, en forma resumida, que el uso de herramientas tecnológicas: "le da a los alumnos la oportunidad de solidificar y ampliar sus conocimientos matemáticos [...] y pueden estimular el aprendizaje de las matemáticas en los alumnos" Guin, y Trouche. (1999)

Así, investigar y documentar los procesos cognitivos que muestren los alumnos mientras resuelven problemas o actividades con apoyo de la tecnología, como el software dinámico, resulta una tarea que puede ayudar a identificar y analizar las ventajas y / o desventajas que el uso de dichas herramientas representa en el aprendizaje de la geometría.

Se discutió en el ICME (11th International Congress on Mathematical Education) Monterrey México que la incorporación de nuevas tecnologías en la resolución de problemas requiere amplia investigación acerca de los diferentes usos en los procesos de aprendizaje de los estudiantes. En este sentido, "los resultados producidos por las computadoras y sus aplicaciones están cambiando profundamente la forma de desarrollar las matemáticas, la forma de enseñarlas, así como la forma de aprenderlas" Stewart, I. (1990)

Un aspecto relevante en el empleo de la tecnología es el tipo de demostraciones o pruebas que pueden presentar los estudiantes; así, resulta necesario identificar los aspectos que se favorecen al utilizar software dinámico en actividades en la que los alumnos tengan oportunidad de descubrir relaciones, plantear conjeturas, generalizar resultados y utilizar argumentos que justifiquen sus soluciones o resultados.

Las posibilidades que tiene el software dinámico para organizar datos son excepcionales; entre otros, puede ampliar la capacidad del alumno para realizar esta misma tarea en diferentes contextos de resolución de problemas. Pero para que esto se dé en ellos se pregunta ¿Qué influencia tiene el uso de software dinámico en la so- lución de problemas geométricos y la conexión entre lo oral y lo escrito para los alumnos?.¿Cuáles son las ventajas que tienen los comandos con relación a los teoremas, postulados entre otros elementos que se consideran en la geometría plana; de tal forma, que permita conectar estos recursos con otras asignaturas sustentadas en la resolución de problemas para su formación profesional?

Los estudios recientes por investigadores en la disciplina, permiten responder en síntesis los interrogantes anteriores, en éstos se hace énfasis, propiamente en la resolución de problemas, mediante el uso de los recursos informáticos. En las investigaciones, también se contemplan actividades que estimulan la experimentación, el descubrimiento, el planteamiento y justificación de conjeturas con tecnología en el proceso de enseñanza aprendizaje de la matemática. Todos estos argumentos deben ser de suma importancia en los objetivos de una clase. Así, es importante analizar el impacto o relevancia del uso de software dinámico en ambientes de resolución de problemas donde los estudiantes de forma consistente, exhiban sus formas de razonamiento.

Santos y Moreno (2001) resaltan que "el uso sistemático de la tecnología con el tiempo se va convirtiendo en una herramienta poderosa para que los estudiantes le den sentido a la información, que realicen conjeturas y que examinen diferentes estrategias en la resolución de problemas" 1 . Santos, M. y Moreno, L,(2001) p. 10 En particular, interesa analizar el tipo de representaciones, las conjeturas y argumentos que utilizan los alumnos durante el desarrollo de las actividades.

El estudio diagnóstico realizado en esta investigación, en el que se indagaron aspectos sobre el proceso de enseñanza aprendizaje de la geometría en el programa de ingeniería mecánica en la Universidad del Norte de Barranquilla Colombia, permitió asumir, que a pesar de los importantes esfuerzos que en esta dirección se realizan, se manifiestan insuficiencias vinculadas con:

${ }^{1}$ Santos, M. y Moreno, L, (2001) Proceso de Transformación del Uso de Tecnología en Herramientas para Solucionar Problemas de Matemáticas por los Estudiantes. Seminario Nacional de Formación de Docentes: Uso de Herramientas Tecnológicas en el Aula de Matemáticas. Colombia: Ministerio de Educación Nacional, No 1. p.10 
1. Bajo nivel en la integración y aplicación de los conocimientos apropiados al escenario laboral profesional de desempeño.

2. Limitaciones en la consulta de variadas fuentes de información y autogestión del conocimiento necesario para el cumplimiento de las funciones profesionales en la asignatura Geometría Plana en especial con los conceptos básicos asociados a la construcción de un cuadrado, el concepto de isósceles en un triángulo y otros temas fundamentales de la geometría.

3. Limitaciones en la identificación de problemas, valoración de alternativas y propuestas de soluciones creativas e innovadoras, y en la diferenciación de tesis, que aunque conocen los recursos para enfrentar el problema se les dificulta utilizarlos.

4. Las limitaciones en la comunicación (oral, escrita y gráfica) al insertarse en equipos de trabajo propios de la actividad ingenieril

El análisis del proceso de enseñanza aprendizaje de la geometría que se realiza en la carrera de Ingeniería Mecánica, para la búsqueda y valoración de las causas de tales insuficiencias, permitió precisar que, se manifiestan en la comprensión de problemas donde se dan afirmaciones sin justificación, lo que generalmente conduce a encontrar soluciones sin ningún sustento matemático, predominando en ellos el argumento "se supone que", lo que limita lograr que el alumno generalice el problema, por lo que la conexión de la solución al entorno se torna difícil, donde se evidencia una contradicción en su fase externa entre los contenidos asimilados por los alumnos y sus potencialidades para aplicarlos en situaciones concretas.

Lo anterior permitió determinar el problema científico de investigación siguiente: ¿Cómo potenciar el proceso de enseñanza aprendizaje de la geometría en los estudiantes de Ingeniería Mecánica a través del uso de un software dinámico?

La actualidad del problema radica en que, su solución se inserta en el perfeccionamiento que se lleva en las universidades, a partir de garantizar la contextualización del proceso de enseñanza aprendizaje, surgen de proyectos que dinami- cen las actividades y su reproducción en medios informáticos, se muestran la extensión de temas que se pueden trabajar con esta herramienta y en especial la aplicabilidad de los problemas en el campo laboral para el futuro ingeniero, dada la necesidad de que los egresados de estas carreras satisfagan las exigencias que plantean las esferas sociales y productivas en relación con las transformaciones que se suceden en el entorno laboral nacional e internacional.

El presente trabajo de investigación se rigió a partir del siguiente objetivo: Elaborar un modelo didáctico, y un procedimiento para su introducción a la práctica, que contribuya al perfeccionamiento del proceso de enseñanza aprendizaje de la geometría en los alumnos de Ingeniería Mecánica. Este objetivo direcciona el campo de acción, con el uso de software dinámico en el proceso de enseñanza aprendizaje de la geometría en los estudiantes de Ingeniería Mecánica.

Para guiar el proceso de investigación, se propuso la siguiente la hipótesis descriptiva: Un modelo didáctico para la conjetura operacional sustentado en la contradicción interna entre la comprensión de problemas de la geometría sintética, y la solución de los mismos mediado por un conjunto de actividades, un procedimiento con sus correspondientes indicaciones metodológicas si permite la solución de problemas con el uso de un software dinámico y así contribuir al proceso enseñanza aprendizaje de la geometría en los alumnos Ingeniería Mecánica.

Para cumplir con el objetivo y dar respuesta a la anterior hipótesis se definieron las tareas científicas siguientes:

Valorar el proceso de enseñanza aprendizaje de la geometría a través de la resolución de problemas, en los estudiantes de Ingeniería Mecánica.

Fundamentar el uso de software dinámico en el proceso de enseñanza aprendizaje de la matemática.

Diagnosticar la situación existente en el proceso de enseñanza aprendizaje de la geometría en los estudiantes de primer semestre de Ingeniería en la Universidad del Norte.

Diseñar un modelo didáctico dirigido a perfeccionar el proceso de enseñanza aprendizaje de la geometría en carreras técnicas. 
Elaborar un procedimiento que permita la introducción en la práctica del modelo.

Someter a criterios de expertos el modelo para determinar su factibilidad.

Implementar parcialmente en la práctica la propuesta para valorar sus potencialidades.

\section{Metodología}

La investigación se fundamenta en la dialéctica materialista y toma elementos de los paradigmas cuantitativos y cualitativos, y se triangulan los resultados, concepciones y fuentes.

Métodos teóricos, en el análisis y crítica de fuentes, fundamentado en los métodos del pensamiento lógico y en su interrelación, tomados como procedimientos: análisis-síntesis, inducción-deducción y lo histórico-lógico; para analizar la multiplicidad de fuentes utilizadas en esta investigación, como vía para la valoración de hechos, ideas, tendencias y concepciones.

Método hermenéutico, en estudio interpretativo de la pluralidad de conceptos, categorías, proyecciones textuales y parlamentos esgrimidos en torno al tema de investigación; y en la reconstrucción y crítica de fuentes. Se recurre además a la propensión del enfoque de la hermenéutica dialéctica en pos del desarrollo de la contradicción.

La modelación y el método sistémico - estructural, en la elaboración del modelo didáctico, y el procedimiento cuyas partes se encuentran en estrecha interrelación.

Observación simple, del proceso de enseñanza aprendizaje de la geometría, en el programa de Ingeniería Mecánica, para constatar el uso de software dinámico y sus resultados.

Enfoque de observación participante como parte del accionar del investigador a lo largo de los cinco años de la investigación y que es un valioso auxiliar en el diagnóstico y la validación de la tesis.

Intervención parcial en la práctica, para valorar las potencialidades de la propuesta, con métodos empíricos en el proceso de aplicación parcial en la práctica de la propuesta, que permita involucrar a los participantes en su propia transformación.
Entrevistas a estudiantes para obtener información del estado actual del problema con un amplio número de participantes, en el proceso. El criterio de expertos, a través del método Delphi, para determinar la pertinencia del modelo didáctico y su procedimiento.

La novedad se encuentra en la formulación y justificación de conjeturas en la resolución de problemas de la geometría con estudiantes de una carrera no matemática, y en este sentido potenciar su aprendizaje a través de las facilidades que tiene el software dinámico para visualizar y conceptualizar sobre los cuerpos geométricos.

El aporte teórico de la tesis se manifiesta en la concepción de un modelo didáctico que se sustenta en la contradicción entre la comprensión de problemas de la geometría sintética, y la solución de los mismos que al enfocarlos a través de la génesis instrumental permitió establecer factores mediadores que pueden acelerar la solución de la misma.

Un aporte práctico está contenido en el procedimiento en el cual se establecen las etapas, acciones, preguntas apoyadas en consideraciones heurísticas e indicaciones metodológicas para guiar un conjunto actividades que deben permitir la concreción en el proceso enseñanza aprendizaje de la geometría a través de la resolución de problemas mediante el uso de software dinámico

\section{FUNDAMENTOS TEORICOS A PARTIR DEL USO DEL SOTFWARE DINAMICO EN LA ENSEÑANZA APRENDIZAJE DE LA GEO- METRIA EN INGENIERIA}

La fundamentación del uso de software dinámico en el proceso de enseñanza.-aprendizaje de la geometría, en él se describe la importancia de los recursos informáticos. Además, se analizan los aportes teóricos de investigadores reconocidos en la disciplina; los cuales permiten orientar y valorar esta investigación a través de la resolución de problemas de Geometría en la Ingeniería Mecánica. Además, la valoración del proceso con una visión retrospectiva y el sustento epistemológico lo que conllevará a potenciar el proceso de enseñanza aprendizaje de los estudiantes de Ingeniería Mecánica mediante el uso del software dinámico. 
Fundamentación del uso del software en el proceso de enseñanza aprendizaje de la matemática

Entrar a discutir científicamente los procesos de enseñanza aprendizaje de la matemática, es entrar sin lugar a dudas hacer parte de una de las disciplinas más polémicas en la literatura científica, debido a los diferentes enfoques con que se manejan los programas curriculares y los métodos que cada sistema imparte.

Las investigaciones recientes discutidas en los congresos mundiales de matemáticas tales como el ICME (11th International Congress on Mathematical Education), el PME (International Group For the Psychology of Mathematics Education) y los RELME (Reunión Latinoamericana de Matemática Educativa) y otros, permiten comprometer al docente cada vez más con una práctica educativa coherente y permisible en el dominio de los contenidos por parte de los estudiantes. En este sentido se han hecho investigaciones recientes en donde el uso de técnicas avanzadas, ha logrado ampliar y solidificar el conocimiento.

Es por ello que dado el avance tecnológico, en el ámbito educativo, es posible plantearse preguntas como la siguiente: ¿Cómo influye la tecnología en el proceso de enseñanza aprendizaje de las matemáticas?. Para tratar de responder esta pregunta se han realizado varios trabajos relacionados con diversas tecnologías, enfocados con este complejo proceso. Por ejemplo, (Kieran y Guzmán, 2003) afirman que "en la investigación sobre el uso de tecnología en la enseñanza aprendizaje de las matemáticas se está realizando de dos distintas maneras: la primera está relacionada con el diseño de actividades, y la segunda, con el desarrollo de éstas" p. 148

Lo anterior, permite comprometer al docente a mejorar su práctica educativa, y en este sentido ser más responsable de los malos resultados que registra el ICFES (Instituto Colombiano para el Fomento de la Educación Superior) en torno al proceso de enseñanza aprendizaje de las matemáticas, en este orden de ideas, la exposición de las diferentes plenarias debatidas en el reciente ICME 11 deja claro que en América Latina y algunos países subdesarrollados registran malos resultados en el área de matemáticas, y más cuando no tienen acceso pleno al uso de las nuevas tecnologías fácil de adquirir.

Experiencias de investigación obtenidas directamente por el investigador de esta tesis, en uno de los centros de importancia mundial el Centro de Investigaciones y Estudios Avanzados (Cinvestav) del Instituto Politécnico Nacional de México (IPN) permiten puntualizar que cualquier tipo de software diseñado para mejorar los procesos de enseñanza aprendizaje de la matemática, facilita tanto al maestro como al alumno, la comprensión; por ejemplo de un teorema, la interpretación de los conceptos matemáticos y en su orden, la contextualización de lo visual con lo abstracto, siempre y cuando el docente a través de la actividad, logre una sinergia entre estudiante y tecnología, esta relación pedagógica se logra mediante un modelo didáctico de conjetura operacional, como se aprecia en la figura anterior.

Los Resultados de la valoración del modelo didáctico y la puesta en práctica del procedimiento, está estructurado en dos epígrafes; el primero se ocupa de la determinación de la factibilidad del modelo y el procedimiento por medio del criterio de experto, el segundo está dirigido a la valoración de la propuesta a partir de su instrumentación parcial en la práctica, tal como se muestra en los siguientes resultados:

Caracterización de variables en condiciones iníciales: descrita todas las etapas de la actividad se diagnosticó el conocimiento de los alumnos, a través de una prueba que permitió medir el número de respuestas por alumno, el número de conceptos correctamente manejados, el número de respuestas correctas con software dinámico y finalmente el número de conceptos con software, cada uno de estos aciertos estaba representado por una variable (ver tabla 1)

El número de respuestas incorrectas, supera al número de respuestas correctas en cada una de las variables establecidas, la razón se debe en gran parte que los alumnos no tienen claras las definiciones básicas y conceptos de la geometría que se enseña en el bachillerato, esto repercute que el número de conceptos con software dinámico es de cero, como se aprecia en las siguientes descripciones: 
Figura 2. Descripción de procedimiento en condiciones iníciales

\section{Descripcion del procedimiento Con papel y lapir}

En el triangulo $A B C$ el lado $A B$ es igual al lado $A C$ y mide $5 \mathrm{~cm}$ $4 \mathrm{~cm}$ entonces el area del tríangulo es lomismo oll el perimetro osea $\frac{A=A B \times B C}{2}=5 \times 4=10 \mathrm{~cm}$.

Caracterización de variables establecidas en condiciones finales: esta fase incluye el diagnóstico permanente; es decir, está presente en todas las fases del modelo, su realización y análisis permitió conocer el estado actual de la situación existente respecto al interés de los alumnos de ingeniería para mejorar en cuanto al conocimiento de algunos conceptos básicos de la geometría, al considerar que el uso de software dinámico, puede servir como un aliado en las clases de matemática, se valora la comprensión de los problemas de la geometría, lo que favorecen aspectos de la contradicción con las potencialidades para aplicarlos en situaciones concretas tal como se muestra en la tabla 1.

La caracterización de variables establecidas en condiciones finales presenta seis columnas que contiene la caracterización porcentual de cada una de las variables que fueron medidas después de haber incluido el software dinámico en las clases de geometría durante 20 secciones de trabajo. En la columna tres se observa un rendimiento del 57\% comparado con el número de respuestas correctas dadas en condiciones iníciales; es decir, de las 63 respuesta correctas dadas por los 24 alumnos en condiciones iníciales aumento en 240 respuestas correctas en condiciones finales, y así sucesivamente el rendimiento se observa en la columna que contiene la variable establecida; la columna seis muestra un rendimiento de $0 \%$ a $79 \%$, permite asegurar el proceso de instrumentación en condiciones finales. Aquí los alumnos relacionan los conceptos

Tabla 1. Caracterización de variables establecidas en condiciones finales

\begin{tabular}{|l|c|c|c|c|c|}
\hline \multirow{2}{*}{ Calificación } & $\begin{array}{c}\text { ítems del examen } \\
\text { variables }\end{array}$ & $\begin{array}{c}\text { (X): Número } \\
\text { de respuestas }\end{array}$ & $\begin{array}{c}\text { (Y):Número } \\
\text { de conceptos }\end{array}$ & $\begin{array}{c}\text { (Z):Número de } \\
\text { R con S }\end{array}$ & $\begin{array}{c}\text { (Q): Número } \\
\text { de C. con S }\end{array}$ \\
\hline Total & 1056 & 312 & 216 & 312 & 216 \\
\hline Correctas & 879 & $240=77 \%$ & $184=85 \%$ & $285=91 \%$ & $170=79 \%$ \\
\hline Incorrectas & 177 & $72=23 \%$ & $32=15 \%$ & $27=9 \%$ & $46=21 \%$ \\
\hline
\end{tabular}


Figura 3. Descripción de procedimiento en condiciones finales

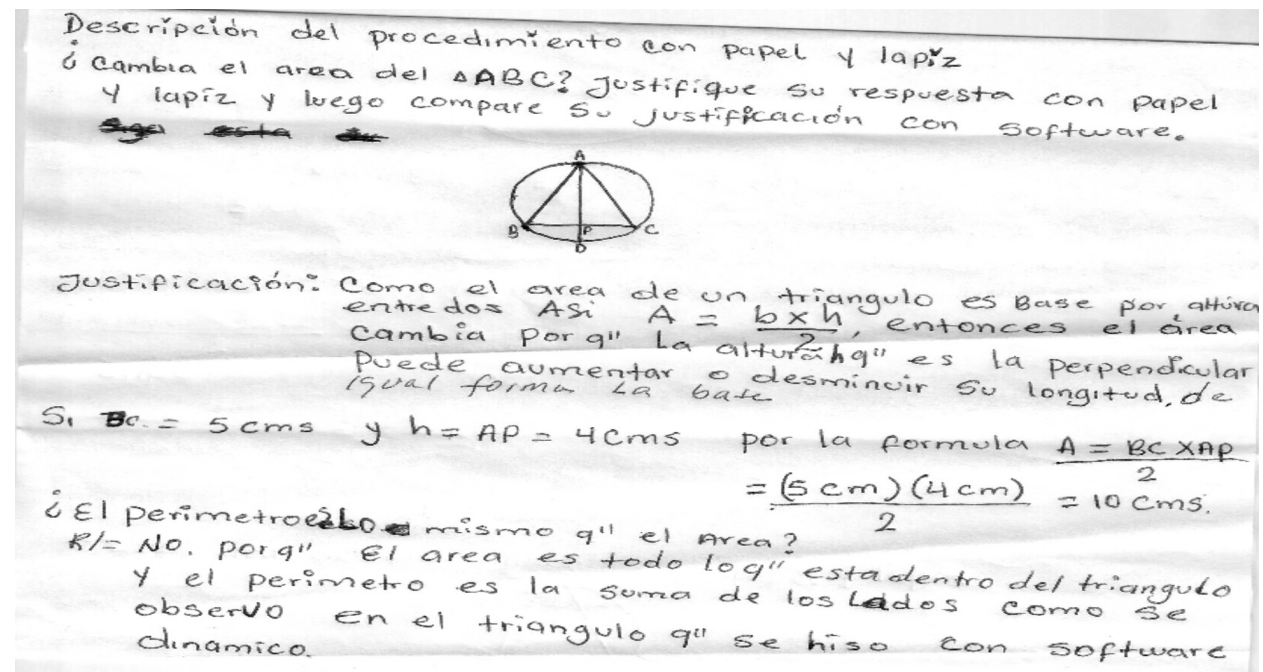

básicos de la geometría con los elementos que contiene el software para plantear y argumentar sus conjeturas como se indica en las siguientes descripciones:

\section{Preguntas que orientaron la investigación}

- ¿Qué aspectos del quehacer matemático se favorecen cuando los alumnos emplean, sistemáticamente, el software dinámico en sus experiencias de aprendizaje? particularmente, ¿qué tipo de conjeturas formulan ellos, al trabajar actividades de resolución de problemas cuando emplean, sistemáticamente, el software dinámico? Dentro de los aspectos del quehacer matemático que se favorecieron con el uso sistemático del software dinámico se encuentran: i) la investigación en configuraciones, en la mayoría de las actividades los grupos buscaron relaciones; ii) el trabajo con casos particulares, al menos dos participantes tuvieron esta tendencia al resolver los problemas planteados y otros, ocasionalmente, intentaron resolver problemas a partir de casos específicos; iii) la búsqueda de contraejemplos que en varias ocasiones utilizaron los alumnos para descartar conjeturas y iv) argumentos que permitan justificar y comunicar resultados.

- ¿Cuál es el proceso que muestran los alumnos al convertir el artefacto "softwa$\mathrm{re}^{\prime \prime}$ en una herramienta matemática de trabajo? Con el uso sistemático del software dinámico los alumnos pudieron llevar a cabo ideas que sólo era posible desarrollar en este tipo de ambientes; un claro ejemplo es el procedimiento realizado por el estudiante " $A$ " en la Actividad 4 para verificar que dos segmentos son paralelos, solución en la que se contemplan ideas relacionadas con rectas paralelas y que media con el instrumento.

Valoración a través del criterio de expertos del modelo didáctico para favorecer el proceso enseñanza aprendizaje de la geometría en carreras de ingeniería

Se presenta a continuación los resultados de expertos consultados sobre el modelo, en detalle se hace una descripción del juicio intuitivo emitido por cada uno de ellos, para así tener un consenso de opiniones, también se detalla la valoración de la instrumentación puesta en práctica. El método de criterio de expertos, consiste en la utilización sistemática del juicio intuitivo de un grupo de expertos para obtener un consenso de opiniones informadas (Rodríguez y Concepción, 2004).

Para sacar adelante la valoración tanto del 
modelo, como del procedimiento conjetura operacional mediante el uso de software dinámico en la enseñanza aprendizaje de la geometría en carreras de ingeniería, primeramente se realizó la encuesta (ver Anexo 9) para la selección de los expertos, la misma se aplicó a 30 profesionales para decidir los expertos a partir del coeficiente de competencia (ver Anexo 10), de los profesionales encuestados. De ellos, fueron seleccionados 27 de los cuales 24 tienen coeficiente de competencia alto y 3 tienen coeficiente de competencia medio.

Esta selección de expertos fueron tomados en cuenta, ya que el coeficiente de competencia medio de esta población es alto $(0,82)$. Los mismos imparten cátedra en las diferentes carreras de ingeniería de la Universidad del Norte de Barranquilla Colombia; se caracterizan por tener de 10 a 45 años de experiencia como promedio en la docencia y/o la investigación. Además, el autor valoró esta experiencia a partir de la producción intelectual de cada participante y su participación en eventos científicos con relación al tema de tesis. De los expertos seleccionados 17 son doctores y 10 ostentan título de máster, algunos con títulos de ingeniería. El alto nivel académico de los docentes se debe en parte a que la Universidad del Norte tiene convenios académicos con universidades de Alemania, México, España, Estados Unidos entre otros países, lo que repercute un alto número de doctores egresados.

El procedimiento para consensuar criterio de expertos se realizó en dos fases de trabajo, las cuales les permitieron emitir sus criterios y valoraciones con base al modelo. Cada experto, de manera individual, se pronunció con respecto a los referentes puntos; de esta forma se pudo determinar la concordancia de los aspectos que se sometieron a consideración En dicha encuesta, ellos emitieron sugerencias que permitió perfeccionar la propuesta

Para someter la propuesta a consideración de los expertos se elaboró una tabla que contiene las fases y componentes del modelo didáctico para el perfeccionamiento del proceso de enseñanza aprendizaje de la geometría en carreras de ingeniería. En la tabla 4 los expertos debían marcar en una escala de cinco categorías, la calificación que consideraban tenía cada aspecto, las categorías evaluativas empleadas fueron: muy adecuado (MA), bastante adecuado (BA), adecuado (A), poco adecuado (PA) y no adecuado (NA).

Los argumentos expuestos por los expertos con relación a los interrogantes planteados en la encuesta, concuerdan en las siguientes consideraciones:

Como se aprecia en la tabla anterior, se observa la valoración de la consulta a los expertos encuestados, en la cual fue sometido a su criterio los componentes del modelo didáctico para perfeccionamiento proceso enseñanza aprendizaje de la geometría en carreras de ingeniería, y se obtiene como resultado:

> Que los componentes de la primera fase son considerados por los expertos como muy aceptables

> Que todos los componentes de la segunda fase son considerados por los expertos como bastante aceptable

> Para la tercera fase se aprecia lo siguiente:

- En la formación e implementación del software, la necesidad de incluir el software dinámico es calificada como bastante aceptable y en la otra alternativa como muy aceptable.

- Para el caso de la génesis instrumental, la creatividad en la instrumentalización e instrumentación es muy aceptable.

- En cuanto a los elementos mediadores 
Tabla 2. Argumentos expuestos por los expertos con relación a los interrogantes planteados

\begin{tabular}{|c|c|c|c|c|c|c|c|}
\hline \multicolumn{3}{|c|}{$\begin{array}{l}\text { Modelo didáctico para el perfeccionamiento del proceso de enseñanza } \\
\text { aprendizaje de la geometría en carreras de ingeniería }\end{array}$} & \multirow[t]{2}{*}{ MA } & \multirow[t]{2}{*}{ BA } & \multirow[t]{2}{*}{ A } & \multirow[t]{2}{*}{ PA } & \multirow[t]{2}{*}{ NA } \\
\hline FASES & \multicolumn{2}{|l|}{ COMPONENTES } & & & & & \\
\hline Primera & \multicolumn{2}{|c|}{ Fundamentos, epistemológicos, psicológicos y pedagógicos } & SI & - & - & - & - \\
\hline \multirow{2}{*}{ Segunda } & \multirow{2}{*}{$\begin{array}{l}\text { Diagnóstico que emerge de la } \\
\text { caracterización de variables }\end{array}$} & \multirow{2}{*}{$\begin{array}{l}\text { Condiciones iníciales antes } \\
\text { de aplicar software dinámico } \\
\text { Condiciones finales despues } \\
\text { de aplicar software } \\
\end{array}$} & - & SI & - & - & - \\
\hline & & & SI & - & - & - & - \\
\hline \multirow[t]{2}{*}{ Tercera } & \multirow{2}{*}{$\begin{array}{l}\text { Desenvolvimiento del } \\
\text { software dinámico a través } \\
\text { de la génesis instrumental } \\
\text { (resolución) }\end{array}$} & $\begin{array}{l}\text { Proceso } \\
\text { Instrumentalización }\end{array}$ & - & SI & - & - & - \\
\hline & & Proceso de instrumentación & Si & - & - & - & - \\
\hline \multirow{5}{*}{ Cuarta } & \multirow{2}{*}{$\begin{array}{ll}\text { Elementos } & \text { mediadores } \\
\text { transversales }\end{array}$} & Mediación instrumental & - & SI & - & - & - \\
\hline & & Motivación profesional & Si & - & - & - & - \\
\hline & \multirow{3}{*}{$\begin{array}{l}\text { Factores que integran la } \\
\text { conjetura operacional }\end{array}$} & Flexibilidad & $\mathrm{Si}$ & - & - & - & - \\
\hline & & Fluidez asociativa & - & SI & - & - & - \\
\hline & & Fluidez figurativa & SI & - & - & - & - \\
\hline QUINTA & \multicolumn{2}{|c|}{$\begin{array}{l}\text { Concreción en la práctica: } \\
\text { Procedimiento conjetura operacional a través del IECO }\end{array}$} & Si & - & - & - & - \\
\hline
\end{tabular}

la orientación investigativa acorde con la mediación instrumental es evaluado como muy aceptable y la relación entre mediación y motivación como bastante aceptable.

- Para los dos restantes factores que integran la conjetura operacional con su indicador de efectividad concuerdan en que los mismos son muy aceptables.

> La cuarta fase es catalogada en los resultados cuantitativos como muy aceptable (ver Anexo 12)

Lo explicado hasta el momento en lo referente a la valoración cuantitativa de la consulta realizada permite llegar a la conclusión general de que los expertos concuerdan en que todos los aspectos consultados son pertinentes en el modelo propuesto.
En la valoración cualitativa dada por los expertos, referente al modelo didáctico para el perfeccionamiento del proceso de enseñanza aprendizaje de la geometría en carreras de ingeniería en cuanto a las fases y componentes del modelo que deberían ser incluidas o eliminadas, modificados $\mathrm{u}$ otros aspectos que los mismos consideran de importancia para tener en cuenta en el modelo se tiene: (ver tabla 3).

Luego de la primera fase, con motivo a las sugerencias efectuadas por los expertos, se reestructuraron algunos elementos del modelo y se consideró la función y la relación de cada uno de los momentos que conforman el modelo conjetura operacional y así se pudo lograr un mejor consenso de lo que se aplicaría posteriormente. 
Tabla 3. Observaciones cualitativas de la consulta a los expertos

\begin{tabular}{|c|c|}
\hline Recomendación del experto & Observación del autor compartida con el experto \\
\hline $\begin{array}{l}\text { 1. En la segunda fase comparar los } \\
\text { resultados obtenidos antes de } \\
\text { utilizar el software dinámico con } \\
\text { los resultados obtenidos después } \\
\text { de implementarlo }\end{array}$ & $\begin{array}{l}\text { La recomendación es esencial para destacar los avances en } \\
\text { cuanto al proceso de enseñanza aprendizaje de la geometría, } \\
\text { mediante un indicador de efectividad planteado por el autor. }\end{array}$ \\
\hline $\begin{array}{l}\text { 2. Proponer la } \quad \text { mediación } \\
\text { instrumental y la motivación } \\
\text { profesional como elementos } \\
\text { mediadores transversales }\end{array}$ & $\begin{array}{l}\text { En esta investigación la mediación instrumental a través de } \\
\text { cabrí, cumple un factor importante como mediador entre lo } \\
\text { cognitivo y lo instrumental, esto se logra a partir de la motivación } \\
\text { entre recurso, alumno y profesor }\end{array}$ \\
\hline $\begin{array}{l}\text { 3. Incluir el software dinámico en los } \\
\text { contenidos programáticos }\end{array}$ & $\begin{array}{l}\text { En cuanto a la inclusión de incorporar los recursos informáticos } \\
\text { en los planes de estudio, la Universidad del Norte e insiste en } \\
\text { la incorporación de algún recurso informático en los planes de } \\
\text { estudio para superar el bajo rendimiento de los alumnos en } \\
\text { matemáticas }\end{array}$ \\
\hline $\begin{array}{l}\text { 4. Incluir en la cuarta fase el elemento } \\
\text { de flexibilidad }\end{array}$ & $\begin{array}{l}\text { El autor considera que el principio de flexibilidad, permite } \\
\text { integrar y dinamizar los dos elementos trasversales con la } \\
\text { fluidez figurativa y asociativa como factores importantes de la } \\
\text { conjetura operacional }\end{array}$ \\
\hline $\begin{array}{l}\text { 5. Resaltar este tipo de } \\
\text { procedimientos heurísticos }\end{array}$ & $\begin{array}{l}\text { El autor esta de acuerdo que el modelo didáctico, a pesar } \\
\text { que fue diseñado para el perfeccionamiento del proceso de } \\
\text { enseñanza aprendizaje de la geometría en carreras técnicas, } \\
\text { también puede funcionar en otras asignaturas de matemáticas. }\end{array}$ \\
\hline $\begin{array}{l}\text { 6. El IECO como soporte del } \\
\text { procedimiento heurístico }\end{array}$ & $\begin{array}{l}\text { Se entiende que esta es una recomendación a nivel académico } \\
\text { que pone de manifiesto el indicador de efectividad de la } \\
\text { conjetura operacional al poner en práctica el procedimiento }\end{array}$ \\
\hline $\begin{array}{l}\text { 7. Divulgar y poner en práctica la } \\
\text { investigación }\end{array}$ & $\begin{array}{l}\text { Desde que se empezó la investigación, las directivas mostraron } \\
\text { interés en apoyar el proyecto. }\end{array}$ \\
\hline
\end{tabular}

Los resultados cuantitativos de la encuesta aplicada a los expertos, arrojan aspectos favorables en cuanto a fases que conforman el modelo, así como lo relativo al procedimiento e indicador de efectividad lo valoran de muy adecuado (MA) o bastante adecuado (BA). Entre los aspectos que con mayor frecuencia se observan, está la flexibilidad, la fluidez figurativa, seguido del proceso de instrumentalización y la concreción práctica a través del indicador de efectividad en sus dos condiciones y así sucesivamente los demás aspectos presentes en el modelo.
Entre las consideraciones sugeridas por los especialistas que se tuvieron en cuenta para mejorar el procedimiento se encuentra: (a) Argumentar y profundizar en los fundamentos del modelo, principalmente aquellos que permitan fortalecer los contenidos de otras asignaturas con los contenidos de la geometría. (b) Considerar la génesis instrumental como un momento del modelo. (c) Proponer actividades, que evidencien la construcción y justificación de las conjeturas, con las relaciones entre las categorías propuestas. (d) Resaltar estadísticamente el indicador de efectividad para conocer 
Tabla 4. Tabla de resultados de los valores absolutos de la encuesta aplicada a los expertos

\begin{tabular}{|c|c|c|c|c|c|c|c|}
\hline \multicolumn{8}{|c|}{ VALORACIÓN CUANTITATIVA DEL MODELO DIDACTICO } \\
\hline \multicolumn{3}{|c|}{$\begin{array}{l}\text { Modelo didáctico mediante software dinámico para el perfeccionamiento } \\
\text { de la enseñanza-aprendizaje de la geometría en Ingeniería }\end{array}$} & MA & BA & A & PA & NA \\
\hline FASES & \multicolumn{7}{|c|}{ COMPONENTES } \\
\hline \multirow{3}{*}{ Primera } & \multicolumn{2}{|c|}{ Fundamentos epistemológicos, psicológicos y pedagógicos } & 10 & 16 & 1 & 0 & 0 \\
\hline & \multirow{2}{*}{$\begin{array}{c}\text { Diagnóstico que emerge } \\
\text { de la caracterización de } \\
\text { variables }\end{array}$} & $\begin{array}{l}\text { Condiciones iníciales antes de } \\
\text { aplicar software dinámico }\end{array}$ & 9 & 11 & 1 & 0 & 0 \\
\hline & & $\begin{array}{l}\text { Condiciones finales después de } \\
\text { aplicar software }\end{array}$ & 15 & 14 & 4 & 0 & 0 \\
\hline Segunda & \multirow{4}{*}{$\begin{array}{c}\text { Desenvolvimiento del } \\
\text { software dinámico a través } \\
\text { de la génesis instrumental } \\
\text { (resolución) } \\
\text { Elementos mediadores } \\
\text { transversales }\end{array}$} & Proceso de Instrumentalización & 18 & 7 & 2 & 0 & 0 \\
\hline \multirow{3}{*}{ Tercera } & & Proceso de instrumentación & 14 & 11 & 2 & 0 & 0 \\
\hline & & Mediación instrumental & 10 & 17 & 0 & 0 & 0 \\
\hline & & Motivación profesional & 17 & 9 & 1 & 0 & 0 \\
\hline \multirow{3}{*}{ Cuarta } & \multirow{3}{*}{$\begin{array}{c}\text { Factores para la conjetura } \\
\text { operacional como } \\
\text { elementos mediadores }\end{array}$} & Flexibilidad & 20 & 7 & 0 & 0 & 0 \\
\hline & & Fluidez asociativa & 16 & 10 & 1 & 0 & 0 \\
\hline & & Fluidez figurativa & 20 & 7 & 4 & 0 & 0 \\
\hline Quinta & \multicolumn{2}{|c|}{$\begin{array}{l}\text { Concreción en la práctica: } \\
\text { Procedimiento conjetura operacional a través del IECO }\end{array}$} & 18 & 9 & 0 & 0 & 0 \\
\hline
\end{tabular}

el impacto del software dinámico en la caracterización de variables dadas en las dos condiciones del diagnóstico.

En la consulta realizada, se observa un consenso de los expertos sobre el procedimiento heurístico para favorecer la formulación y justificación de conjeturas dada por los alumnos, por esta razón fue necesario plantear actividades que involucran al software dinámico en la solución del problema y comparar la solución con lápiz y papel

Al analizar y comparar los límites de categorías de cada uno de los ítems que se encomendaron, las que más destacan los expertos son: muy adecuado y bastante adecuado; se obtiene como sugerencia, integrar más el procedimiento heurístico con asignaturas afines con la geometría, tales como la aplicación de la misma en la enseñanza aprendizaje del cálculo, ecuaciones diferenciales, etc. Es decir, para lograr en este sentido una expresión gráfica que integre la conjetura operacional mediante software dinámico con los componentes didácticos y sus relaciones para que signifiquen las dimensiones de este proceso, además de alcanzar una mejor comprensión y nivel de síntesis del modelo conjetura operacional.

Valoración de los resultados obtenidos en la instrumentación práctica del modelo didáctico

Con el propósito de ofrecer una información detallada de la implementación del modelo didáctico conjetura operacional se brinda una valoración cualitativa y cuantitativa del desarrollo del proceso durante su aplicación. En el mismo aparecen los principales resultados obtenidos en el desarrollo de la experiencia. Además, se describe el procedimiento empleado en la investigación a través del indicador de efectividad y se detallan las características principales de los alumnos que participaron en el estudio así como se indican los pasos metodológicos de cada actividad que se desarrolló 


\section{Participantes en la investigación}

En la investigación participaron veinte cuatro alumnos, ocho mujeres y dieciséis hombres (con edades entre dieciséis y treinta años); los alumnos cursaban segundo semestre en ingeniería en la Universidad del Norte de Barranquilla, Colombia. Los participantes del estudio fueron, en su mayoría, alumnos que aprobaron su semestre anterior con calificación mínima en el curso de matemáticas (sus calificaciones promediaron 3.3 en el curso "Algebra y Trigonometría").

Antes del desarrollo de este estudio algunos alumnos no conocían algún tipo de software dinámico, aunque algunos habían interactuado con un software distinto a lo exigido, otros conocían Cabrí; así, fue posible iniciar las primeras sesiones de trabajo relacionadas con las herramientas básicas de construcción, los alumnos se familiarizaron con los comandos del software, les resultó interesante recurrir al "arrastre" de puntos, realizar cálculo de medidas con la calculadora del software y efectuar ensam- bles de construcciones básicas. Esto les permitió expresar consideraciones como estas:

Los escritos presentados por los alumnos, permitieron considerar que el software dinámico les brinda no solo la oportunidad de dinamizar el problema, sino que les abre la posibilidad de acercarse a la definición precisa después de varios intentos " $B-C$ tiene que mantenerse perpendicular a $A-D$, por lo que solo se puede subir y bajar".

Además, cabe resaltar que el software dinámico, le facilita otros elementos pedagógicos que a simple vista, con papel y lápiz, se le dificulta describir; por ejemplo en la invariabilidad del área del triángulo afirman que: "el área se mantiene igual porque la base y la altura son directamente proporcionales, la base disminuye pero la altura aumenta de allí que la altura se mantiene en igual proporción". Los alumnos describen la conjetura de la compensación, lo que aumenta en la altura disminuye en la base. Aunque no dan la respuesta acertada, si hay unas categorías y leyes que constituyen el principio del conocimiento. Ver figura, a lápiz

\section{Figura a lápiz}

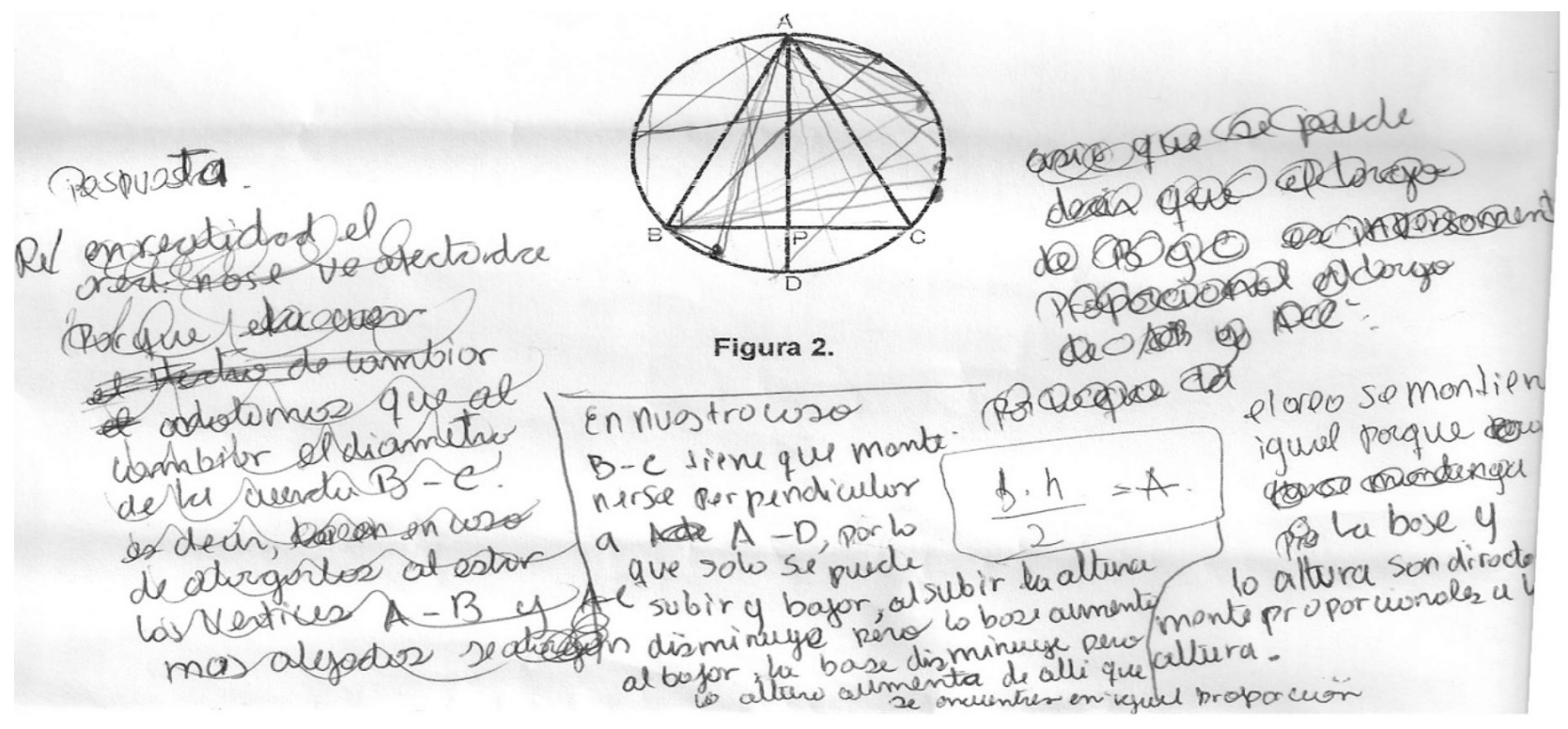


Escenarios • Vol. 14, No. 2, Julio - Diciembre de 2016, págs. 31-44

Se trae a colación el concepto de didáctica, de Concepción M. R. y Rodríguez, F. (2004) la didáctica como ciencia, además de tener delimitado su objeto de estudio: el proceso de enseñanza aprendizaje, tiene un sistema de categorías y leyes que constituyen el fundamento teórico metodológico para instruir y educar, construido con base en la investigación científica pedagógica, el cual se nutre además, de las ciencias como la Pedagogía, Psicología, Filosofía, Lógica, y de la naturaleza de las ciencias concretas que se estudian en la escuela que enriquecen y singularizan a las mismas.

\section{Conclusiones}

Las nuevas tecnologías, como el software, la calculadora gráfica con capacidad de procesamiento simbólico-algebraico, el uso del Internet, son recursos fundamentales que no deben pasar inadvertidos en los programas de estudios de las instituciones educativas de todos los niveles de la educación.

Es por ello que en el proceso de enseñanza aprendizaje de la matemática de las diferentes carreras, en especial los programa de ingeniería y de otras carreras análogas, como matemática y las licenciatura de cada una de las ciencias básicas, obliga a una permanente actualización y una estrecha interrelación entre los contenidos de las diversas disciplinas.

Lo anterior conllevó a una continua transposición didáctica desde las teorías y enfoques del software dinámico hacia su implementación en la práctica mediante la construcción y solución de problemas de carácter geométrico.

No se trata solo de resolver problemas de manera tradicional, basados en la memoria y los algoritmos existentes desde la antigüedad, sino que es más que una simple recomendación de incorporar las nuevas tecnologías en el aula. Es decir, se constituye en una obligación social generacional.
Todo lo anterior justifica con creces, que ejercicios científicos como el que se acaba de mostrar en el campo de acción del proceso de enseñanza aprendizaje de la geometría, deben ir más allá de la herencia de Euclides y las curiosidades de Eratóstenes.

Dentro de esta concepción, la conjetura operacional se constituye en una expresión dinámica y flexible para elevar la calidad en el proceso de enseñanza aprendizaje de la geometría cuando el alumno logra pasar de la instrumentalización al proceso de instrumentación. Tal sinergia se logró, en el momento que los elementos mediadores transversales como la mediación instrumental y la motivación profesional se integran con fluidez y flexibilidad para formar los factores del modelo presentado y la operacionalización del mismo a través de un procedimiento.

\section{Referencias}

Álvarez de Z., C. (1995): Metodología de la investigación científica. Centro de Estudios de Educación Superior "Manuel F Gran", Santiago de Cuba, Cuba.

Álvarez de Z, C. (1995) la Pedagogía como ciencia o Epistemología de la Educación Edit. Pueblo y Educación, 3ra Edición, Ciudad de la Habana-Cuba, p. 6

Álvarez de Z., C. y García, R. (1997): La contradicción dialéctica como invariante para la reestructuración del Proceso Docente Educativo. Revista Cubana de Educación Superior, Vol. XXII, No 2:21-26, Ciudad de La Habana, Cuba.

Álvarez de Z., C. (1999): La Escuela en la Vida. Edit. Pueblo y Educación, 3ra Edición, Ciudad de la Habana, Cuba.

Álvarez de Z., C. (2000): Metodología de la investigación científica. Cómo se modela la investigación científica (en soporte electrónico). La Habana, Cuba.

Concepción M. R. Y Rodríguez, F. Metodolo- 
gía de la Experiencia Pedagógica de Avanzada. En Revista CITMA. Holguín, 2004

Gómez-Chacón, I. Ma (1995): Juegos de estrategia. Apuntes IEPS, $\mathrm{n}^{\circ}$ 55, Madrid: Narcea:134-156.

Gómez-Chacón, I. M ${ }^{a}$ (1998): Matemáticas y contexto. Apuntes IEPS, $n^{\circ}$ 64, Madrid: Nancea:45-56.

Guin and Trouche (2002): Mastering by the teacher of the instrumental genesis in CAS environments: necessity of instrumental orchestrations. ZDM, vol. 34 (5).

Guin, y Trouche, L. (1999) The Complex Process of Converting Tools loto Mathematical

Instruments: The Case of Calculators. International Journal of Computers Jor Mathematical Learning, 3, p.195

Kieran, C. y Guzmán, J. (2003) The Spontaneous Emergent of Elementary Number-Theoretic Concepts and Techniques in Interaction with Computing Technology. In Proceedings of the 2003 Joint Meetinf of PME an PMENA, Neil A. Paterman, Barbara J. Doughert and Joseph Zilliox (Eds) vol. 3, p. 148

Lizarazo, C., (2005): Exploraciones de los alumnos de nivel medio superior mediante el uso de la TI-92 en la solución de sistemas de ecuaciones lineales y no lineales de $2 \times 2$. Tesis de maestría publicada. Departamento de Matemática Educativa, Cinvestav IPN México: 7- 32.

Lizarazo, C., (2005): El papel de la calculadora TI-92 en la solución de sistemas de ecuaciones lineales y no lineales: estudio con alumnos de nivel medio superior. Revista de Ciencias Básicas Bolivarianas, ISSN 16577450 No.6:7-14.

Santos, M. y Moreno, L, (2001) Proceso de Transformación del Uso de Tecnología en Herramientas para Solucionar Problemas de Matemáticas por los Estudiantes. Seminario
Nacional de Formación de Docentes: Uso de Herramientas Tecnológicas en el Aula de Matemáticas. Colombia: Ministerio de Educación Nacional, No 1. p.10

Concepción M. R. Y Rodríguez, F. Metodología de la Experiencia Pedagógica de Avanzada. En Revista CITMA. Holguín, 2004

Rodríguez, F y Concepción, R. (2004) El método Delphi para el procesamiento de los resultados de encuestas a expertos o usuarios en estudios de mercado y en la investigación educacional. Material Impreso para los Cursos de FesCaribe. Barranquilla. Colombia.

Piaget, J. (1970): Seis Estudios de Psicología. Editorial Planeta-Agostini, Barcelona, 1993.

Polya, G. (1981): Mathematical discovery (combined ed). Wiley, New York.

Polya, G. (1965): Cómo Plantear y Resolver Problemas.Trillas reimpresión 2002, México. Pp.101-102.

Santos, M. y Moreno, L, (2001) Proceso de Transformación del Uso de Tecnología en Herramientas para Solucionar Problemas de Matemáticas por los Estudiantes. Seminario Nacional de Formación de Docentes: Uso de Herramientas Tecnológicas en el Aula de Matemáticas. Colombia: Ministerio de Educación Nacional, No 1. p.10

Stewart, I. (1990) Change. in L. Steen (Ed) On the Shoulders of Giants. New Aproache Numeracy, Washington, DC, USA: National Academy Press. p. 180

Vygotsky,L.S. (1991): Obras Escogidas. Tomo I. Visor. España. Watzlawick, P.; Blanin, J. B. $\mathrm{y}$

Vygotsky, L.S. (1956): Izbranie psiholgicestie issledovanija [Investigación en psicología]. Moscú, APN RSFSR.

Vygotsky, L.S. (1960): Razvitie vyssih psihiceskih funkcil [Desarrollo de las funciones psicológicas superiores]. Moscú, APN RSFSR. 